\title{
Role of fine needle aspiration cytology in breast cancer
} screening

\author{
JOAN LAMB*, T J ANDERSON*, M J DIXON $\dagger$, PAMELAALEVACK $\dagger$ \\ From the Departments of *Pathology and $\dagger$ Clinical Surgery, University of Edinburgh, Scotland
}

SUMMARY In a six year period up to the end of December 1985 fine needle aspiration cytology specimens of the breast were obtained from 562 apparently healthy women invited to participate in a breast cancer screening programme. Of these, 397 had a biopsy and 173 cancers were confirmed histologically. For the diagnosis of cancer, the procedure was less successful than in symptomatic cases. The main factors influencing success were the aspirator, the small size of many cancers, and the occult nature of the lesions seen only on mammography. Retrospective analysis of the figures shows that combining the results of FNA cytology in a triple assessment with physical and mammographical findings for restricted selection means that the number of benign biopsy specimens could be reduced considerably.

Cytology is a well established method of investigating breast lesions in symptomatic patients, but there is little recorded information about its use as part of a well woman screening programme. This paper reports the results of fine needle aspiration (FNA) cytology in invited well women attending a breast screening clinic over a period of six years up to the end of December 1985. In this paper we assess the accuracy of fine needle aspiration cytology in preoperative diagnosis of cancer and its contribution to the selection of cases for biopsy in a screening programme.

\section{Material and methods}

SOURCE OF CLINICAL MATERIAL D

The FNA cytology specimens were taken from invited well women participating in the Edinburgh Breast Screening Project (EBSP) ${ }^{1}$ which forms part of the United Kingdom seven year trial of early breast cancer detection. ${ }^{2}$ The decision to recommend for biopsy, based on clinical and mammographical examination, was taken at a surgical review clinic. As part of the protocol and before any further investigation took place, a clinic assessment category, reflecting the expected biopsy result, was recorded for each invited well woman; this category was for internal clinic guidance. The aspirates were obtained from solid lesions and the cytology assessed as previously described. ${ }^{3}$

Accepted for publication 5 February 1987

\section{PATHOLOGY}

The pathology findings in the biopsy specimens were extracted from the pathology register for the EBSP; the size of any invasive cancer was the maximum diameter of the lesion, as measured macroscopically in the biopsy specimen. A zero size was recorded for non-invasive cancers.

\section{EVALUATION OF FNA RESULTS}

The standard variables of specificity, sensitivity, and predictive value ${ }^{4}$ were applied to this group of cases as the measures of performance of a selection procedure in diagnosing or identifying women most likely to have cancer. Both complete and absolute values were calculated. ${ }^{5}$ Absolute values refer to those cases specifically diagnosed as having malignant cells; complete values relate to the group showing either suspicious or malignant cells.

\section{Results}

In a six year period up to the end of December 1985 562 invited well women from the Edinburgh breast screening clinic had FNA cytology of the breast performed. Of these, 397 women went forward for biopsy and 173 cancers were confirmed by histology. The remaining 165 women continued in the screening programme without biopsy and were followed up for at least 12 months after FNA. Of these 165 , one returned two years later with a cancer in the same breast; and one of the cases with a benign biopsy result was found to have an invasive cancer in the 
contralateral breast four years later. The 397 cases biopsied represent $57 \%$ of the biopsies generated by screening in Edinburgh during those years. The 173 histologically confirmed cancers represent $67 \%$ of the total cancers detected by screening during the same time period.

\section{DIAGNOSIS OF CANCER}

Table 1 shows the correlation of the FNA cytology results with the histology findings for the 397 cases biopsied. Notably, there was a high acellular rate, comprising 130 (33\%) of 397 aspirates. Although most of these acellular aspirates was from benign lesions, 29 came from cases of cancer. In another 55 cases aspirates were termed suspicious, and of these, 35 were found to have cancer. The "suspicious" group in this series was a broad group ranging from highly suspicious, but with perhaps poorly cellular aspirates, to cellular aspirates with atypical cytological features. It was intended to represent a strong indication for further investigation. There was one false positive report. Repeat aspirates were taken in 27 women-12 with cancer, in whom seven aspirate results were converted to positive or suspicious; the more severe result was used for the purposes of compiling the table. Of the 173 cancers, only $87(50 \cdot 3 \%)$ had a positive cytology report. Thus during the six years the overall failure rate for diagnosis of cancer by FNA within this group was high.

Further analysis of the figures for the 173 cancers shows that the proportions of the four categories of reporting vary from year to year (table 2). Fig 1 shows the proportions expressed on an annual basis; 1979 is an incomplete year and is combined with 1980 . There was a noticeable improvement in the results in 1982 , with almost double the percentage of positive results $(65.5 \%)$, compared with $1981(34.3 \%)$, and this improvement was maintained throughout 1983. The numbers of acellular and benign reports in these cancers decreased but they were not eliminated, the lowest levels being in 1982, with acellular at $3.5 \%$ and benign at $10 \cdot 3 \%$. After a decrease in the positive results for 1984 the results improved again in 1985 . There was a relatively high proportion $(12 \cdot 5 \%-29 \cdot 4 \%)$ of cases with "suspicious" reports

Table 1 Correlation of cytology with final histology of biopsies from screening cases

\begin{tabular}{llll}
\hline & \multicolumn{2}{l}{ Histology } & \\
\cline { 2 - 3 } Cytology & Malignant & Benign & Total \\
\hline Positive & 87 & 1 & 88 \\
Suspicious & 35 & 20 & 55 \\
Benign & 22 & 102 & 124 \\
Acellular & 29 & 101 & 130 \\
Total & 173 & 224 & 397 \\
\hline
\end{tabular}

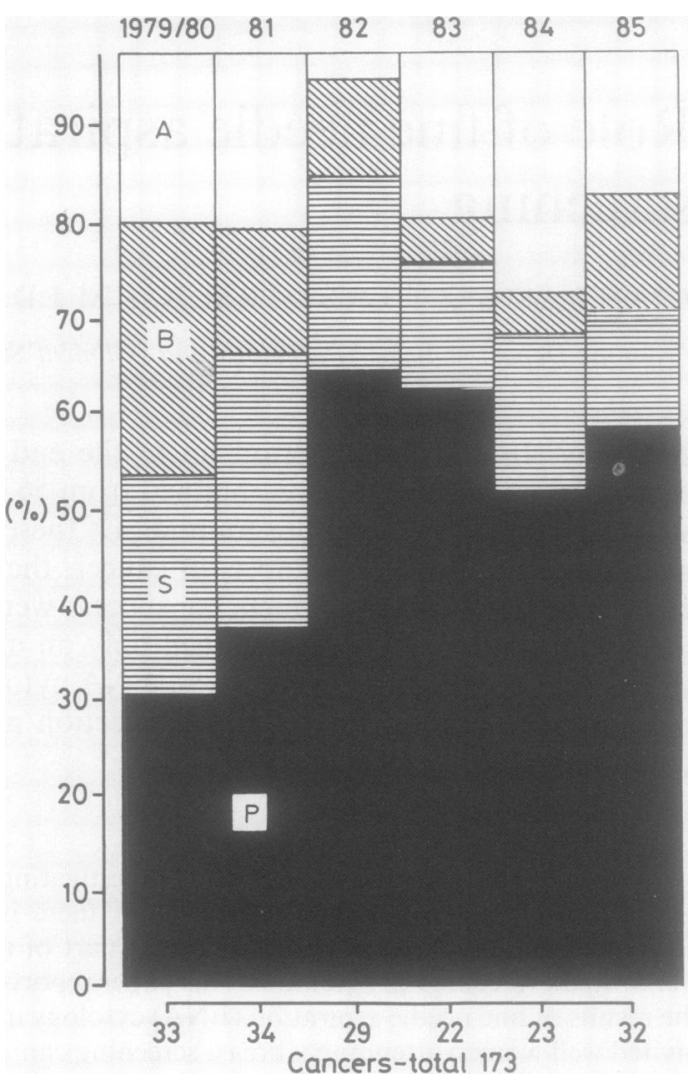

Fig. 1 Cytology results in histologically confirmed primary cancers expressed proportionally on annual basis, with No of cancers given for each year. $P=$ positive (malignant cells present); $S=$ suspicious (indication for further investigation); $B=$ benign (adequate specimen but negative); $A=$ acellular (inadequate specimen).

each year, particularly, but not exclusively, in those years where the number of positive results was low. The years 1982 and 1983 were those for which there was an assigned aspirator (MD) responsible for the aspirations. In 1984 a different aspirator (PL) took over and this changeover period was reflected by an

Table 2 Cytology results for histologically confirmed cancers expressed annually as No of cases

\begin{tabular}{lrrrrrrr}
\hline \multicolumn{7}{c}{ Year of diagnosis } & \\
\cline { 2 - 6 } Cytology & $79 / 80$ & 81 & 82 & 83 & 84 & 85 & Total \\
\hline Positive (P) & 10 & 13 & 19 & 14 & 12 & 19 & 87 \\
Suspicious (S) & 8 & 10 & 6 & 3 & 4 & 4 & 35 \\
Benign (B) & 9 & 4 & 3 & 1 & 1 & 4 & 22 \\
Acellular (A) & 6 & 7 & 1 & 4 & 6 & 5 & 29 \\
Total & 33 & 34 & 29 & 22 & 23 & 32 & 173 \\
\hline
\end{tabular}


Table 3 Clinic assessment category of biopsied cases based on clinical or mammographical examination

\begin{tabular}{|c|c|}
\hline Category & No of biopsies \\
\hline $\begin{array}{ll}1 & \text { Normal } \\
2 & \text { Diffuse benign } \\
3 & \text { Local benign } \\
4 & \text { Suspicious } \\
5 & \text { Malignant }\end{array}$ & $\begin{array}{r}0 \\
6 \\
122 \\
149 \\
116\end{array}$ \\
\hline
\end{tabular}

initial decrease in positive reports and an increase in acellular specimens. The results improved again in 1985. Before 198214 people performed the fine needle aspirations.

Fig 2 shows the correlation of size of the cancers with the cytology results for the 173 cancers. The percentage of cancers identified with positive and positive plus suspicious results decreased with the size of the lesion, particularly for those below $30 \mathrm{~mm}$. One hundred and sixteen $(67 \%)$ of these cancers detected by screening were $20 \mathrm{~mm}$ or less in size. Also included in these figures are 14 occult cancers that were identified by mammography alone. Even so, three of these had a positive, and one, a suspicious cytology report. The group shown in the graph as having zero size are the non-invasive cancers of which seven were in the occult group.

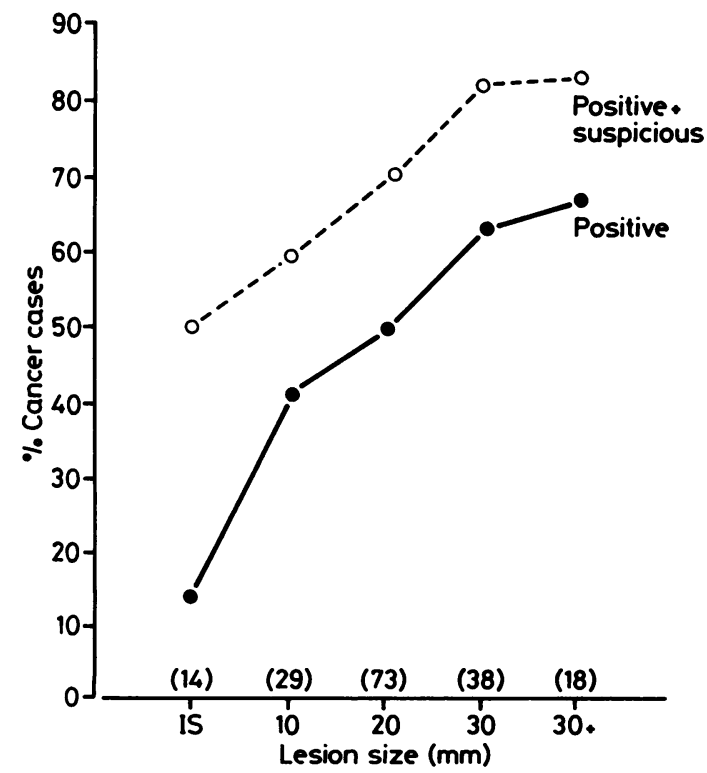

Fig. 2 Percentge of cancers reported as positive cytology $(\bigcirc)$ or positive plus suspicious (O) according to lesion size measured macroscopically on excised tissue. Groups are arranged in $10 \mathrm{~mm}$ size increments except for non-invasive lesions grouped as in situ (15) without specified size. No of cases are given in parentheses.
Table 4 Correlation of clinic assessment category with cytology findings*

\begin{tabular}{lllrrrr}
\hline & \multicolumn{7}{l}{ Clinic assessment category } & \\
\cline { 2 - 6 } Cytology & $I$ & 2 & 3 & 4 & 5 & Total \\
\hline Positive & 0 & 0 & 3 & 23 & 62 & 88 \\
Suspicious & 0 & 1 & 9 & 24 & 19 & 53 \\
Benign & 0 & 5 & 53 & 49 & 14 & 121 \\
Acellular & 0 & 0 & 57 & 53 & 21 & 131 \\
Total & 0 & 6 & 122 & 149 & 116 & 393 \\
\hline
\end{tabular}

*Four cases with clinic assessment unavailable.

These assessments show clearly that size and physical detectability, in addition to the aspirator, have a major influence on the diagnostic success of FNA cytology in the detection of cancer by screening.

\section{SELECTION OF CASES FOR BIOPSY}

Of the 397 cases put forward for biopsy, 224 had benign pathology reports (table 1), and in the context of screening were therefore false positive selections. A clinic assessment category based on combined clinical and mammographical findings was recorded independently at the special review clinic when the decision to biopsy was made. Table 3 shows the distribution of biopsy cases over the five categories; table 4 shows the correlation of the clinic assessment with the cytology reports. The two modalities did not always identify the same cases as being suspicious or malignant. Cytology indicated fewer (141) cases as positive or suspicious than the clinic assessment (265). There was a group of 13 cases where the cytology assessment of positive or suspicious was at variance with the benign clinic assessment. Nine of these 13 cases were histologically confirmed as cancers. A total of 12 cases assigned to clinic category 5 were found to be histologically benign.

Standard measures of performance of a selection procedure-namely, sensitivity, specificity, and pre-

Table 5 Performance measures for cytology alone and combined with clinic category in restricted assessment

\begin{tabular}{|c|c|c|c|}
\hline Measure & $\begin{array}{l}\text { Biopsied } \\
\text { cases } \\
\text { (with FNA) }\end{array}$ & FNA alone & $\begin{array}{l}\text { Combined } \\
\text { restricted } \\
\text { assessment }\end{array}$ \\
\hline $\begin{array}{l}\text { Sensitivity: } \\
\text { Complete } \\
\text { Absolute }\end{array}$ & $\begin{array}{r}100.0 \\
60 \cdot 1\end{array}$ & $\begin{array}{l}70.5 \\
50 \cdot 3\end{array}$ & $\begin{array}{l}99 \cdot 4 \\
74 \cdot 5\end{array}$ \\
\hline Specificity & & $90 \cdot 6$ & $51 \cdot 8$ \\
\hline $\begin{array}{l}\text { Predictive value: } \\
\text { Complete } \\
\text { Absolute }\end{array}$ & $\begin{array}{l}43 \cdot 6 \\
89 \cdot 7\end{array}$ & $\begin{array}{l}85 \cdot 3 \\
98 \cdot 9\end{array}$ & $\begin{array}{l}63.0 \\
91.5\end{array}$ \\
\hline $\begin{array}{l}\text { Selected for } \\
\text { biopsy: }\end{array}$ & 397 & 141 & 278 \\
\hline Cancers detected: & 173 & 122 & 172 \\
\hline
\end{tabular}


dictive value were calculated for cytology alone and for the clinic assessment category combined with cytology. For these purposes two values-absolute and complete-were used. Absolute value referred to only positive (malignant) results regarded as "test" positive; complete referred to positive and suspicious results combined as "test" positive. Table 5 shows the retrospective application of these assessments to our group of 397 biopsy specimens in terms of performance. The complete sensitivity was high (maximal), but the absolute value was low, with the reverse being true of the complete and absolute predictive values. In contrast, fine needle aspiration cytology alone had a low sensitivity in this screening group but a high predictive value (complete and absolute). Therefore in practical terms of selection, use of fine needle aspiration alone in this group of women would have indicated biopsy in only 141 , and detected 122 of the 173 cancers.

Use of cytology combined with clinic assessment category for selection maintained a high sensitivity (both absolute and complete) and increased the predictive value. Of great practical relevance is the possible saving in the number of cases selected for biopsy, reduced from 393 to 278 , and the number of cancers that would have been detected (172). The one cancer that could have been missed in this combined selection process was occult.

\section{Discussion}

FNA cytology of the breast has been used in Edinburgh for several years, and evidence of its value in diagnosis has already been presented. ${ }^{36}$ These reports emphasise, firstly, the importance of the aspiration procedure, where an assigned aspirator with aptitude and commitment can achieve absolute diagnosis in over $80 \%$ of all cases of cancer; and secondly, that a lesion below $20 \mathrm{~mm}$ in size is less likely to be detected. The same influences have continued to be prominent in the present analysis of cases from breast cancer screening. Absolute sensitivity is the measure of success in the diagnosis of cancer by FNA, and reported values range from $88 \cdot 2 \%{ }^{7}$ to $65.9 \%{ }^{8}$ The lower value was achieved only in the "best years" of our present series. Evidently, reduced size of the lesion will remain an obstacle to reaching high levels of diagnostic success in the screening cases, which is unsatisfactory, as the aim of screening is to identify cancers when they are small. This is clearly happening in the Edinburgh project, and the role of the aspirator therefore assumes major relevance.

It could be argued, however, that in screening the principal aim is not precise diagnosis of cancer but rather the ability to select cases with the greatest likelihood of developing or having occult cancer. The screening exercise usually comprises two stages; an initial broad selection, with the opportunity for fuller evaluation at the second stage. The influence of such selection procedures is reflected in the ratio of benign to malignant diagnoses in those referred for biopsy from the second stage screen. A four-fold range in values, from $0 \cdot 5: 1$ to $2: 1$, was noted in a recent review of the European projects, ${ }^{9}$ and it was 5.4:1 in the North American report. ${ }^{10}$ The European projects included three series which used FNA cytology in second stage screening, but separate evaluation of its role was not reported. In Edinburgh such assessment can be made and shows clearly that FNA cytology contributes substantially to a retrospective and restricted selection, when combined with clinic assessment category; the yield of cancer is then maximised while maintaining a greater than $50 \%$ reduction in benign biopsy specimens. It is acknowledged that referral for biopsy in screening may be influenced by several other factors not detailed here. The surgical policy adopted for symptomatic women to biopsy all palpable discrete lumps may be followed. There is also an awareness that many of the changes detected at screening are subtle and may not be perceived by a second examiner at a separate procedure for aspiration. Indeed, truly occult lesions, such as the one missed in the restricted triple assessment, require needle localisation biopsy ${ }^{11}$ unless a stereotactic device is available for the cytology to achieve adequate levels of sensitivity. ${ }^{12}$

The performance measures obtained in this analysis refer to a non-consecutive series representing $57 \%$ of biopsies generated by screening and may not truly reflect the results from the full spectrum of such a programme. They also do not represent the sensitivity, specificity, and predictive value of the overall screening procedure; they do, however, provide information that is relevant to the optimal use and interpretation of results. In particular, appreciation of the factors influencing success will assist decisions on whether to repeat the procedure or to defer the decision to biopsy. The role of the cytologist is assumed to be a constant factor in the present series, where examination was virtually restricted to two individuals who frequently conferred, in particular with cases at the borderline of positive and suspicious designation. The proportion of reported cases allotted to the suspicious category varies from $3 \cdot 1 \%^{7}$ to $24 \cdot 4 \%^{8}$ and signifies the variation in reporting policies of cytologists. It is worth noting that the $20 \%$ termed suspicious for the screening series was double that found in our larger series of symptomatic cases. ${ }^{6}$ The most likely explanations for this include poor representation of malignant cells in the specimen as a consequence of size and occult nature, as well as the tendency of screening cancers to be well differentiated or of special type ${ }^{13}$ 
rendering unequivocal cytological distinction of malignancy more difficult.

The problem of false positive breast cytology reports has been discussed previously. ${ }^{614}$ Although the need to maintain quality control is essential and no different from other areas of cytology reporting, the issue is diminished where conservation is the approach being taken in the surgical management of patients with small cancers. Collaboration and communication between those providing and evaluating material is important to avoid errors.

The present analysis has identified factors that must be considered when intending to include FNA cytology in the screening of asymptomatic women for breast cancer. The diagnosis of cancer using FNA cytology is less successful than in symptomatic cases, but identification of "suspicious" cases is almost as good. Although not designed for this purpose, the clinic category has proved valuable combined with FNA, and further analysis of the present series may prove instructive. Adopting a triple assessment of physical examination, mammography, and FNA cytology in the strict selection of women for biopsy at second stage screening has advantages; a considerable reduction in biopsies may be achieved without a parallel reduction in cancer detection by screening. Such assessment depends heavily on locally available expertise.

This work was funded by grants from the Scottish Home and Health Department and the Cancer Research Campaign. We acknowledge the cooperation of Dr Maureen Roberts (clinical director) and the staff at the Edinburgh breast screening clinic, Dr Freda Alexander for advice and comment, and the commitment of Mrs Carolyn Brown who maintained the pathology register. We also acknowledge the cooperation of Professor Forrest, $\mathrm{Mr}$ Chetty, and the staff of the breast unit at Longmore Hospital, Edinburgh.
References

1 Roberts MM, Alexander FE, Anderson TJ, et al. The Edinburgh randomised trial of screening for breast cancer: Description of method. Br J Cancer 1984;50:1-6.

2 United Kingdom Breast Cancer Detection Working Group. Trial of early detection of breast cancer: Description of method. $\mathrm{Br}$ J Cancer 1981;44:618-27.

3 Dixon JM, Anderson TJ, Lamb J, Nixon SJ, Forrest APM. Fine needle aspiration cytology in relationships to clinical examination and mammography in the diagnosis of a solid breast mass. Br J Surg 1984;71:593-6.

4 Galen R, Gambino SR. Beyond normality. In: The predictive value and efficiency of medical diagnosis. New York, John Wiley and Sons, 1975. pp 10-14.

5 Abele JS, Miller TR, Goodson WH, Hunt TK, Hohn DC. Fine needle aspiration of palpable breast masses. A program for staged implementations. Arch Surg 1983;118:859-63.

6 Barrows GH, Anderson TJ, Lamb J, Dixon JM. Fine needle aspiration of breast cancer. Relationship of clinical features to cytology results in 689 primary malignancies. Cancer 1986;58:1493-8.

7 Zajdela A, Ghossein NA, Pilleron JP, Ennuyer A. The value of aspiration cytology in the diagnosis of breast cancer: experience at the Curie Foundation. Cancer 1975;35:499-506.

8 Kline TS, Joshi LP, Hunter SN. Fine needle aspiration of the breast: diagnoses and pitfalls. A review of 3545 cases. Cancer 1979;44:1458-64.

9 Gad A, Thomas BA, Moskowitz M. Screening for breast cancer in Europe: Achievements, problems and future. Recent Res Cancer Res 1984;90:179-94.

10 Baker LH. Breast Cancer Detection Demonstration Project: five year summary report. Ca-A Cancer Journal for Clinicians 1982;32:194-230.

11 Chetty U, Kirkpatrick AE, Anderson TJ, et al. Localisation and excision of occult breast lesions. Br J Surg 1983;70:607-10.

12 Svane G, Silfversward C. Stereotaxic needle biopsy of nonpalpable breast lesions. Cytologic and histopathologic findings. Acta Radiol Diagn 1983;24:283-8.

13 Anderson TJ, Lamb J, Alexander F, et al. Comparative pathology of prevalent and incident cancers detected by breast screening. Lancet 1986;ii:519-22.

14 Lever JV, Trott PA, Webb AJ. Fine needle aspiration cytology. J Clin Pathol 1985;38:1-11.

Requests for reprints to: Dr Joan Lamb, Department of Pathology, University Medical School, Teviot Place, Edinburgh EH8 9AG, Scotland. 